\title{
Genome-wide analysis of differentially expressed profiles of mRNAs, IncRNAs and circRNAs during Cryptosporidium baileyi infection
}

\author{
Guan-Jing Ren, Xian-Cheng Fan, Ting-Li Liu, Sha-Sha Wang and Guang-Hui Zhao*
}

\begin{abstract}
Background: Cryptosporidium baileyi is the most common Cryptosporidium species in birds. However, effective prevention measures and treatment for C. baileyi infection were still not available. Long non-coding RNAs (IncRNAs) and circular RNAs (circRNAs) play important roles in regulating occurrence and progression of many diseases and are identified as effective biomarkers for diagnosis and prognosis of several diseases. In the present study, the expression profiles of host mRNAs, IncRNAs and circRNAs associated with C. baileyi infection were investigated for the first time.

Results: The tracheal tissues of experimental (C. baileyi infection) and control chickens were collected for deep RNA sequencing, and 545,479,934 clean reads were obtained. Of them, 1376 novel IncRNAs were identified, including 1161 long intergenic non-coding RNAs (lincRNAs) and 215 anti-sense IncRNAs. A total of 124 IncRNAs were found to be significantly differentially expressed between the experimental and control groups. Additionally, 14,698 mRNAs and 9085 circRNAs were identified, and significantly different expressions were observed for 1317 mRNAs and 104 circRNAs between two groups. Bioinformatic analyses of gene ontology (GO) and Kyoto Encyclopedia of Genes and Genomes (KEGG) pathway for their targets and source genes suggested that these dysregulated genes may be involved in the interaction between the host and C. baileyi.

Conclusions: The present study revealed the expression profiles of mRNAs, IncRNAs and circRNAs during C. baileyi infection for the first time, and sheds lights on the roles of IncRNAs and circRNAs underlying the pathogenesis of Cryptosporidium infection.
\end{abstract}

Keywords: Differentially expressed profiles, mRNAs, IncRNAs, circRNAs, Cryptosporidium baileyi

\section{Background}

Cryptosporidiosis is one of the most prevalent parasitic diseases in domestic and wild birds, and it has been reported in more than 30 avian species worldwide $[1,2]$. Currently, four species of avian Cryptosporidium, namely Cryptosporidium meleagridis [3], Cryptosporidium baileyi [4], Cryptosporidium galli [5] and Cryptosporidium avium (previous avian genotype V) [6], and 12 genotypes (black duck genotype, Eurasian woodcock genotype, avian genotypes I-IV, VI and goose genotypes I-V) have been

\footnotetext{
* Correspondence: zgh083@nwsuaf.edu.cn

Department of Parasitology, College of Veterinary Medicine, Northwest A\&F University, Yangling 712100, China
}

recognized [7, 8]. Among them, C. baileyi, the most common Cryptosporidium species in birds, has been reported in a wide range of avian hosts worldwide, especially in chickens $[9,10]$. Infection of C. baileyi causes respiratory diseases, presenting symptoms of dyspnoea, coughing, rales and sneezing $[4,10,11]$, and sometimes is associated with high morbidity and mortality, especially in broiler chickens [10], leading to considerable economic losses to the poultry industry [12-14]. In addition, C. baileyi has been observed in the stool of an immunodeficient man, suggesting the zoonotic potential of this species [15]. However, no effective prevention measures or treatments against C. baileyi infection have been developed [16]. 
In the process of attachment, internalization, and formation of parasitophorous vacuoles [17-20], Cryptosporidium molecules (eg. rhoptry protein, microneme protein, dense granules) were released into host cells [21, 22] and the transcriptome of host was dysregulated [23]. Seventy-three genes were upregulated and 74 were downregulated in human HCT-8 cells infected with C. parvum [24], and these differentially expressed genes are associated with cell communication, signal transduction and amino acid metabolic processes. Previous studies have indicated that non-coding RNA (ncRNA) molecules were also involved in host-Cryptosporidium interaction process, and aberrant expressions of microRNAs (miRNAs) were detected in C. parvum infecting epithelial cells $[25,26]$.

Recently, two new classes of ncRNA, namely long non-coding RNAs (lncRNAs) and circular RNAs (circRNAs), were discovered [27, 28], and they were found to play an important role in regulating occurrence and progression of many diseases [29-31]. LncRNAs are a group of non-coding RNAs longer than $200 \mathrm{nt}$ [32] and dysregulated lncRNAs play important roles in progression of cells proliferation, invasiveness and metastasis of breast cancer [33], lung cancer [34] and colorectal cancer [35] through cis- and trans-regulation of gene expressions [36, 37] and miRNA sponges [38, 39]. In the abnormal activity of biological processes, such as cell proliferation, cell motility, immune, or inflammation response from diseases like cardiovascular disorders, inflammatory and autoimmune disease, lncRNAs also participate in the disease processes and contribute to controlling the gene regulatory network of hostpathogen interactions [40-42]. On the other hand, the function of circRNAs remains largely unknown. Previous studies have showed that it could harbor specific miRNAs as miRNA sponges [43-45] to suppresses miRNA activity, resulting in increased levels of miRNA targets, influence the expression of cytokines [46], promote cell cycles and inhibit cell apoptosis to act as a candidate oncogene $[47,48]$. Additionally, both lncRNAs and circRNAs have been identified as effective biomarkers for diagnosis and prognosis of diseases [49, 50]. However, the genome-wide expression and functional roles of IncRNAs and circRNAs in Cryptosporidium infection are unclear. Here, we investigated the expression profiles of mRNAs, IncRNAs and circRNAs associated with $C$. baileyi infection for the first time. Our findings would provide baseline data for developing novel diagnostic and therapeutic targets for avian cryptosporidiosis.

\section{Results}

Identification of IncRNAs in chicken tracheal tissue

A total of 559,574,746 raw reads were produced from the Illumina HiSeq 4000 platform. After abandoned adaptor sequences and low-quality sequences, 545,479,934 clean reads (accounting for $81.82 \mathrm{~Gb}$ ) were obtained, and the percentage of clean reads among raw data in each library ranged from 96.30 to $98.20 \%$. Subsequently, the clean reads were mapped to the latest Gallus gallus reference genome. Firstly, considering the characteristics of lncRNA sequences ( $\geq 200 \mathrm{nt}$, exon count $\geq 2$ ) and their differences from other classes of RNAs (eg. mRNAs, rRNAs, tRNAs, snRNAs, snoRNAs, pre-miRNAs, and pseudogenes), the transcripts were classified into different subtypes using both Scripture beta2 and Cufflinks (v2.1.1). 93.98\% of the identified transcripts $(50,857)$ were known as the reference transcripts, whereas $6.02 \%$ (3061) were the presumed lncRNAs. To further confirm these 3061 lncRNAs, 2823 transcripts were obtained after filter of the low expression transcripts with FPKM $<0.5$. Finally, coding potential analysis was performed using the software $\mathrm{CNCI}$, CPC, Pfam-scan, and PhyloCSF. After being screened by rigorous criteria and four analytic tools, a total of 1376 lncRNAs from the tracheal tissue of chickens were identified and subjected to further analysis (Fig. 1a). The 1376 lncRNAs were composed of 1161 (84.4\%) long intergenic non-coding RNAs (lincRNAs) and 215 (15.6\%) anti-sense lncRNAs (Additional file 1), nevertheless, other types of lncRNAs such as intronic lncRNAs, sense lncRNAs and bidirectional lncRNAs were not detected in our study (Fig. 1b).

\section{Differentially expressed profiles of mRNAs, IncRNAs and circRNAs by RNA sequencing}

The number of overlap mRNAs, lncRNAs and circRNAs in the experimental group compared with the control group is displayed in the Venn diagram, respectively (Fig. 2a-c). A total of 1317 mRNAs and 124 IncRNAs (Additional file 2) were found to be differentially expressed with the $q$-value $<0.05$ and $\mathrm{FDR}<0.05$. Among them, 862 mRNAs were upregulated and 455 were downregulated in three infectious tracheal tissues compared with the controls (Fig. 3a). Meanwhile, 58 and 66 lncRNAs were upregulated and downregulated, respectively (Fig. 3b). According to the criteria of fold change $>2.0,656$ mRNAs were upregulated and 292 were down-regulated. Similarly, 53 and 57 lncRNAs were up-regulated and down-regulated. For circRNAs, 104 remarkably differentially expressed genes (fold change $>2.0, p$-value $<0.05$ and FDR $<0.05$ ) between two groups were identified (Additional file 2). Among them, 65 circRNAs were up-regulated and 39 were downregulated (Fig. 3c).

In order to further understand the similarities among tracheas of chickens at the transcriptomic level, the data of all the differentially expressed genes were used for cluster analysis. The heat map clearly showed that the experimental group and the control group were separately clustered together (Fig. 4). The expression patterns 
a

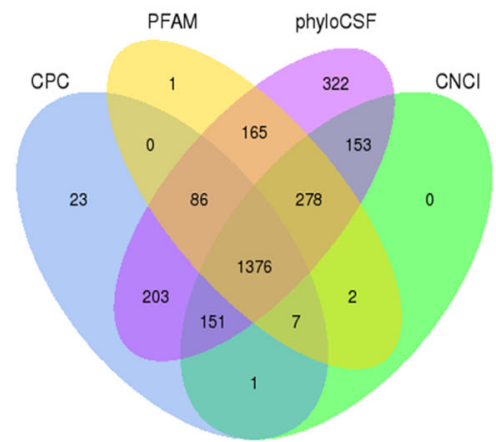

b IncRNA classification

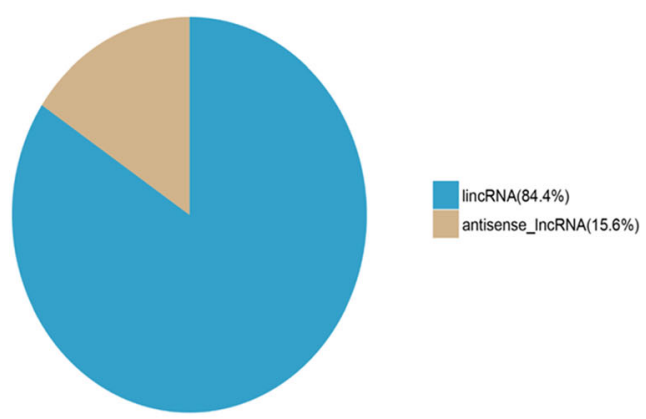

Fig. 1 Screening and classification of the candidate IncRNAs in chicken trachea transcriptome. a Venn diagrams of coding potential analysis according to strict criteria. Four tools (CPC, CNCl, PFAM, and PhyloCSF) were used to analyze the coding potential of IncRNAs. Those simultaneously shared by four analytical tools were designated as candidate IncRNAs and used in subsequent analyses. b Classification of the two subtypes of IncRNAs

of lncRNAs and circRNAs between the experimental group and the control group were distinguishable. These data suggested that the expressions of lncRNAs and circRNAs in chicken tissues infected with C. baileyi were significantly different from those in non-infected chicken tissues.

\section{Comparison of mRNAs and IncRNAs features}

In this study, a total of 14,698 mRNAs, 1616 annotated lncRNAs and 1376 novel lncRNAs were identified from the tracheal tissues of chickens after infection with $C$. baileyi. To examine the differences among these three kinds of transcripts, comparative analysis of gene structure and sequence conservation was conducted. Our results showed that most lncRNAs contained two or three exons, which was significantly less than that of mRNAs (Fig. 5a). However, a slightly discrepancy was observed in the distribution of transcript length between mRNAs and lncRNAs (Fig. 5b). Compared with mRNAs, a relatively shorter open reading frame (ORF) was one of the main features of most lncRNAs (Fig. 5c). In addition, the majority of lncRNAs were less conserved which was different with mRNAs, although the difference is not statistically significant (Fig. 5d).

Validation of dysregulated mRNAs, IncRNAs and circRNAs Ten mRNAs, ten lncRNAs and six circRNAs were randomly selected from the dysregulated genes to be

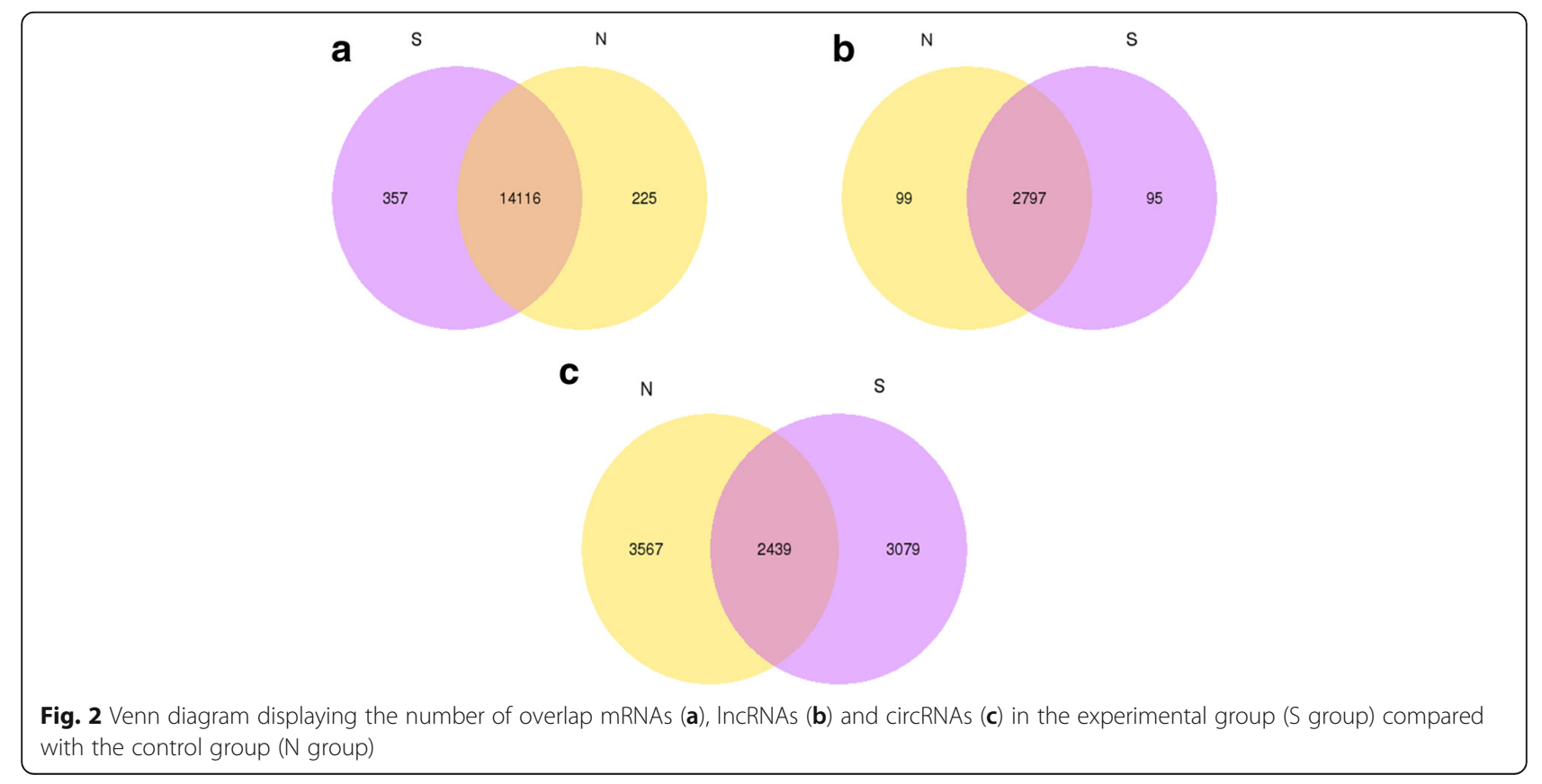




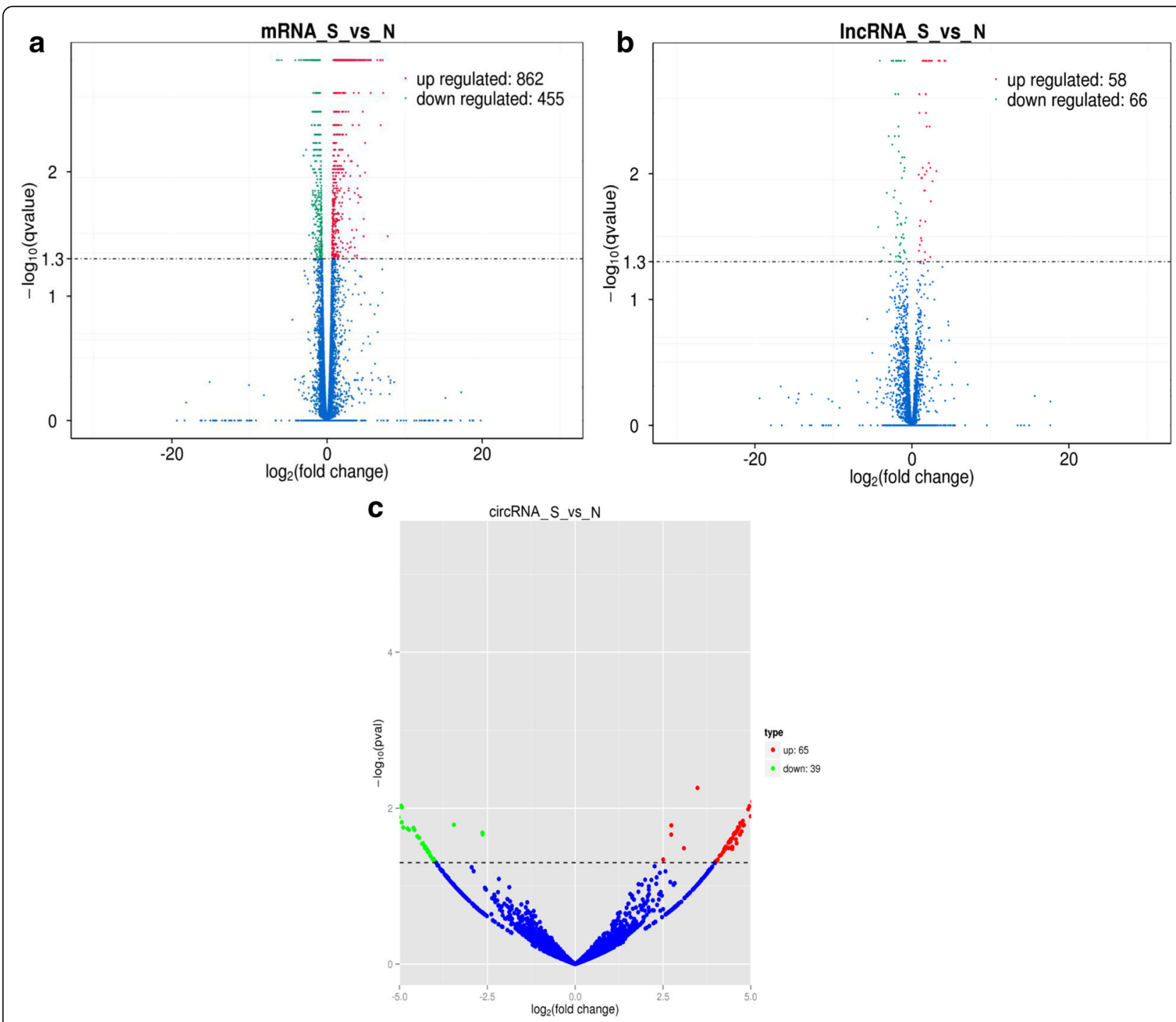

Fig. 3 Differentially expressed mRNAs (a), IncRNAs (b) and circRNAs (c) in the two groups. Volcano plot of the $p$-values as a function of foldchange for mRNAs, IncRNAs and circRNAs indicate the dysregulated genes in the three normal and three infected tissues. Blue dots represent RNAs not significantly differentially expressed ( $p$-value $>0.05$ ) and the other dots represent RNAs differentially expressed $(p$-value $<0.05)$. Up-regulated RNAs were presented as red dots and down-regulated RNAs were presented as green dots

verified by qRT-PCR. It was demonstrated that the expression levels from qRT-PCR data were consistent with the results of RNA sequencing (RNA-seq) (Fig. 6) thus verified the facticity of RNA-seq analysis. Consequently, efficient evidence was provided by this finding that these mRNAs, IncRNAs and circRNAs could be used to investigate the pathogenesis of C. baileyi infection in the future.

\section{Co-expression analysis and target prediction}

Generally, the functions of both lncRNAs and circRNAs were performed by inter-acting with their targets [27, 28]. In our study, the potential cis- and trans-targets were predicted (Additional file 3). The mRNAs $100 \mathrm{~kb}$ upstream and downstream of the lncRNAs were searched for cis analysis, with 2308 lncRNAs that corresponded to 7679 protein-coding genes. The trans analysis of lncRNAs was performed by constructing co-expression networks (Fig. 7) of dysregulated mRNAs and lncRNAs based on expression correlation coefficient (Pearson correlation $>0.95$ or $<-0.95$ ) (Additional file 3). A total of 14,418 mRNAs and 2570 lncRNAs containing 634,780 relationships were detected. More than one mRNAs were predicted to be regulated by one IncRNA, and one mRNA corresponded to several lncRNAs (Fig. 7).

Previous studies have indicated that miRNA sponge was the common function of circRNAs [43, 44]. However, we have not examined the expression profile of miRNA in 

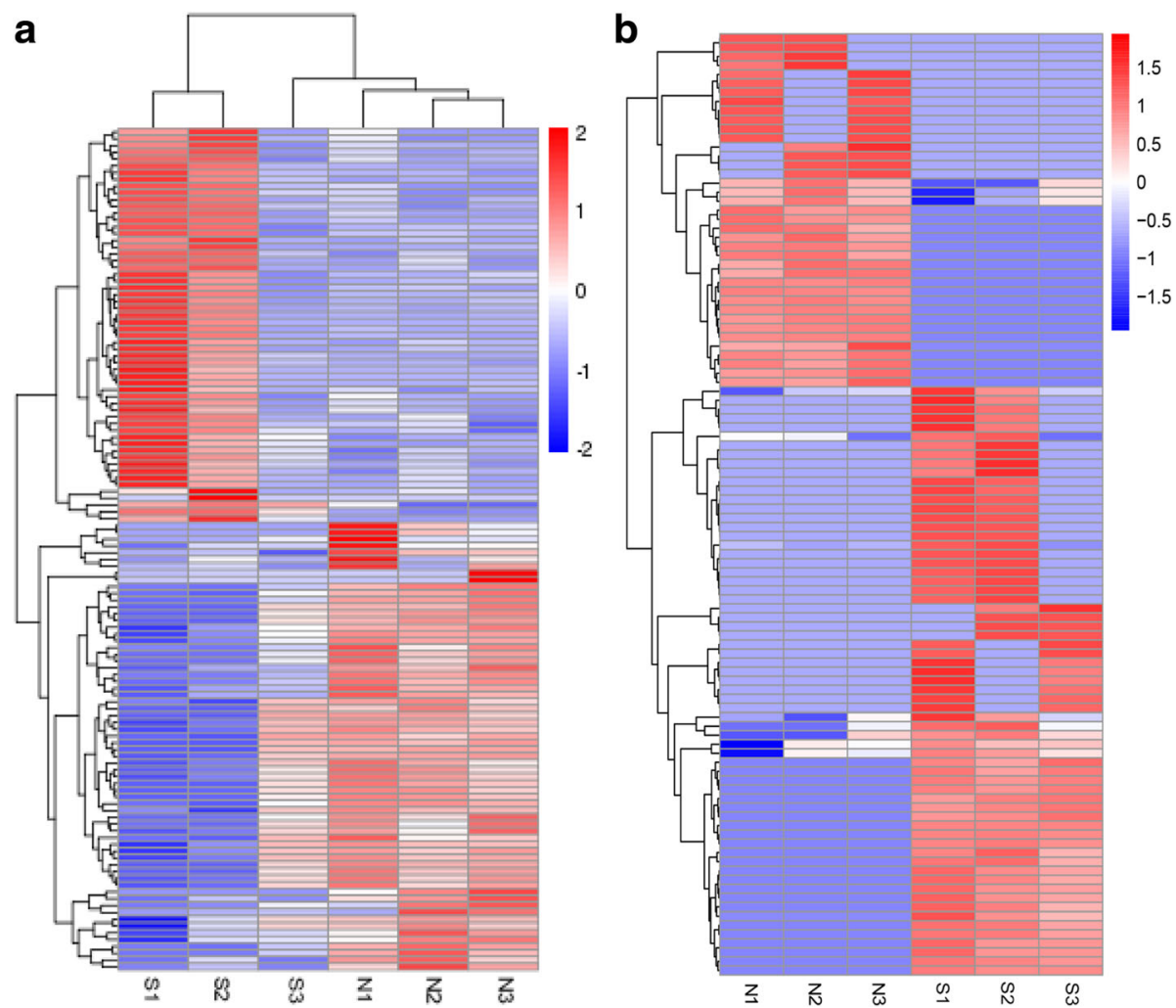

Fig. 4 Heatmap of expression profiles for the IncRNAs (a) and circRNAs (b). Red through blue color indicates high to low expression level. Each column represents one sample, and each row indicates a transcript

chicken tracheas during C. baileyi infection. Therefore, we just predicted the potential miRNA targets based on sequence complementarity in the present study, and the possible interaction relationships between 17,607 circRNAs and the 978 miRNAs in the chicken genome were observed (Additional file 4).

To further predict the function of mRNAs, lncRNAs and circRNAs from tracheal tissue of chickens, the GO (http://www.geneontology.org/) (Additional file 5) and KEGG (http://www.genome.jp/kegg/) pathway analyses (Additional file 6) were performed to analyze the dysregulated mRNAs and the target genes of differentially expressed lncRNAs and the source genes of circRNAs between the two groups.

In the present study, the top 10 results of GO enrichment analysis were selected as the master node of directed acyclic graph (DAG). The DAG of biological process (BP), cellular component (CC) and molecular function (MF) of differentially expressed mRNAs and lncRNAs are shown in Figs. $8 \mathrm{a}-\mathrm{c}$ and $9 \mathrm{a}-\mathrm{c}$, respectively. Based on the GO analysis of differentially expressed mRNAs, the most significantly enriched BP were immune response, immune system process and positive regulation of immune system process, and external side of plasma membrane, cell surface and plasma membrane part were the most enriched CC. Furthermore, microtubule motor activity, motor activity and cytokine receptor activity were identified to be the most significantly enriched MF (Fig. 8d). On the basis of GO analysis of the target genes of differentially expressed lncRNAs, the most significantly enriched BP were immune system process, immune response and leukocyte activation, and the non-membrane-bounded organelle, intracellular nonmembrane-bounded organelle and external side of plasma membrane were the most remarkably enriched CC. In addition, protein binding, purine nucleotide binding and adenyl nucleotide binding were the most significantly enriched MF (Fig. 9d). The most prominent category of gene function will be the focus of future research. Since the numbers of up-regulated and down-regulated circRNAs were small, there were no significant GO terms enriched in the two groups.

As a major public database of pathways, KEGG has been used to determine the significantly enrichment pathways for candidate target genes compared with the entire genome background [51, 52]. The top 20 pathways associated with mRNAs, IncRNAs and circRNAs were showed in an enriched scatter diagram, respectively. For mRNAs, the cytokine-cytokine receptor interaction, intestinal immune network for IgA production 


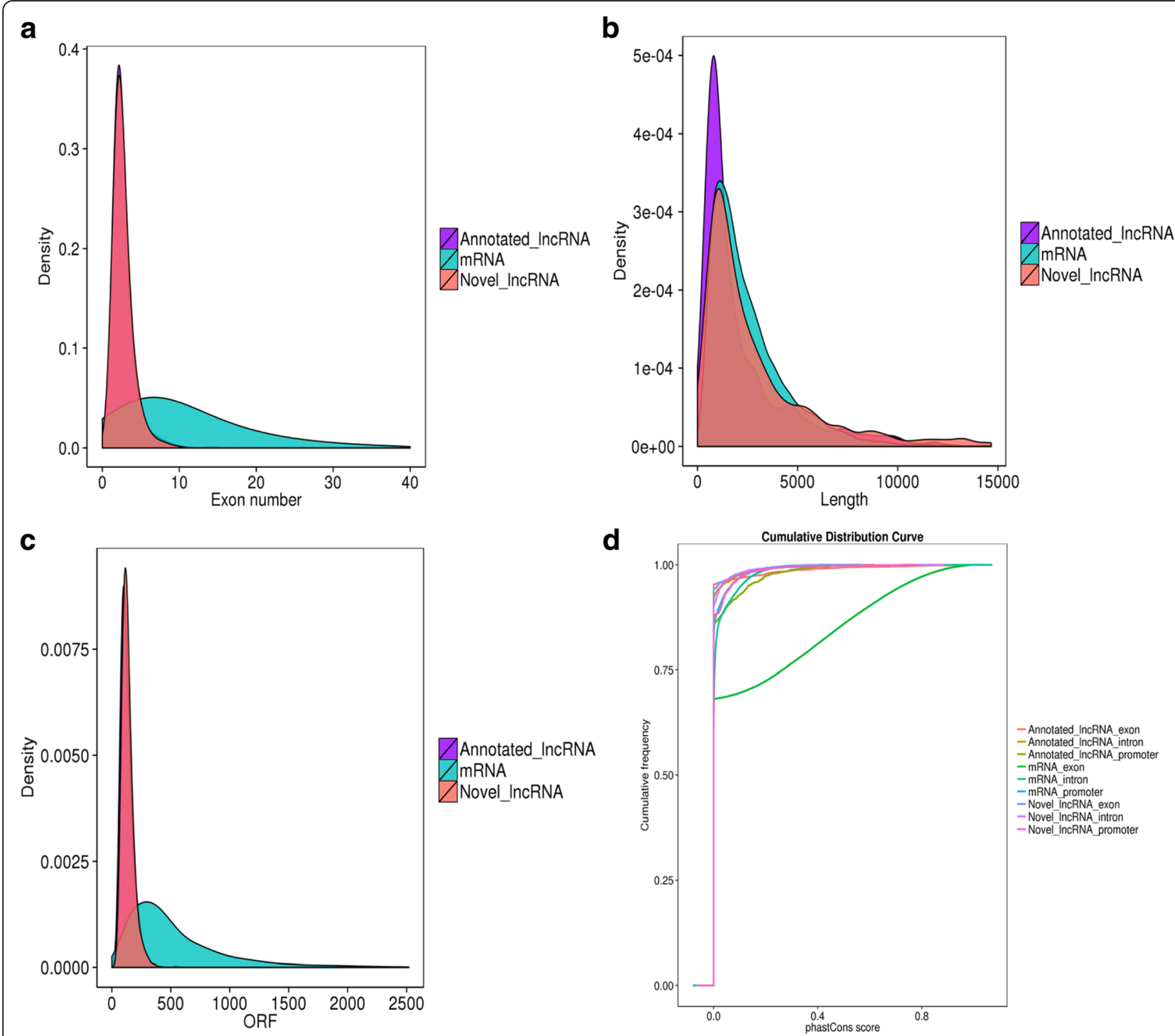

Fig. 5 Comparison of genomic architecture between mRNAs and IncRNAs. a The number of exons in the mRNAs and IncRNAs. In present study, single-exon IncRNAs were filtered out from the chicken genome due to the limitations of the algorithm. $\mathbf{b}$ Distribution of transcript lengths in the mRNAs and IncRNAs. The horizontal axis of indicates the length of transcripts, and the vertical axis represents density. c The number of open reading frames (ORFs) in the mRNAs and IncRNAs. $\mathbf{d}$ Conservation of the sequence in mRNAs and IncRNAs were evaluated using phastCons

and cell adhesion molecules (CAMs) were identified as the top enriched KEGG pathways (Fig. 10a). KEGG pathway enrichment analysis for significantly differentially expressed lncRNAs revealed pathways for intestinal immune network for IgA production, cytokine-cytokine receptor interaction and cell cycle (Fig. 10b). Whereas, the significantly enriched pathways were amino sugar and nucleotide sugar metabolism, tight junction and glycerolipid metabolism for circRNAs (Fig. 10c).

\section{Discussion}

C. baileyi has been considered to be the most common avian Cryptosporidium species worldwide with the broadest host range $[7,53]$. It can reside in the epithelial cells of respiratory tract, causing clinical respiratory disorders [4, 10, 11, 54] in birds (eg. chickens, turkeys and ducks). Considering the distinct morphological and biological features and large oocyst production, $C$. baileyi has been suggested as a model for the characterization of cryptosporidia $[55,56]$. Moreover, $C$. baileyi is able to establish its infection in the mucosal epithelium of a wide variety of organs (eg. respiratory tract, bursa of Fabricius and cloaca) [4, 53], and the trachea is the most common predilection site to present the inflammation and clinical signs. Therefore, the trachea from the experimental and control groups were selected to study the differentially expressed profiles of mRNAs, lncRNAs and circRNAs. The results of our 


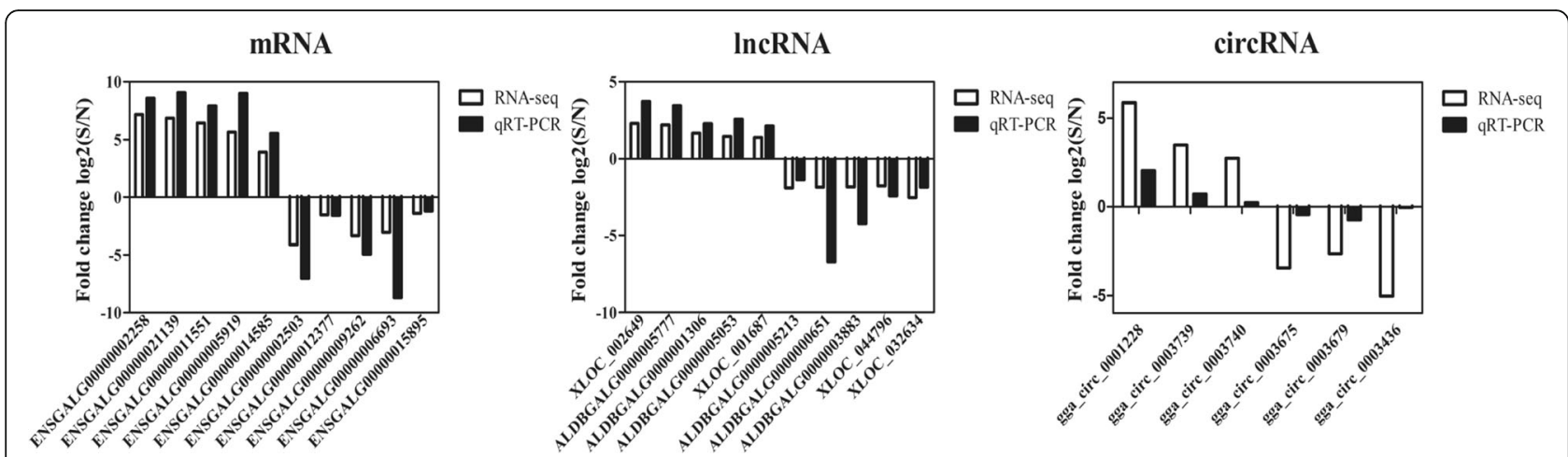

Fig. 6 Comparison of results of RNA sequencing and qRT-PCR. The blank column represents the fold change values obtained from RNA sequencing and the black column displays the fold change values of qRT-PCR. The figures represent the comparison results of mRNAs, IncRNAs and circRNAs from left to right. GAPDH was used as the internal control gene and three biological replicates were used. The expression trends of the results of RNA-seq were consistent with qRT-PCR

study indicated that mRNAs and ncRNAs may play a significant role in the infection process of C. baileyi, representing a potential therapeutic target for C. baileyi infection as revealed by the sequencing analysis.

The chicken genome has been sequenced and assembled using the whole-genome mapping technology in
$2004[57,58]$, with high-quality genome assembly and annotation. The novel mRNAs, IncRNAs and circRNAs obtained in our study greatly improved the annotation of the chicken genome. A total of 14,698 mRNAs were obtained from the tracheal tissue of chickens infected with C. baileyi and 1317 were significantly dysregulated.

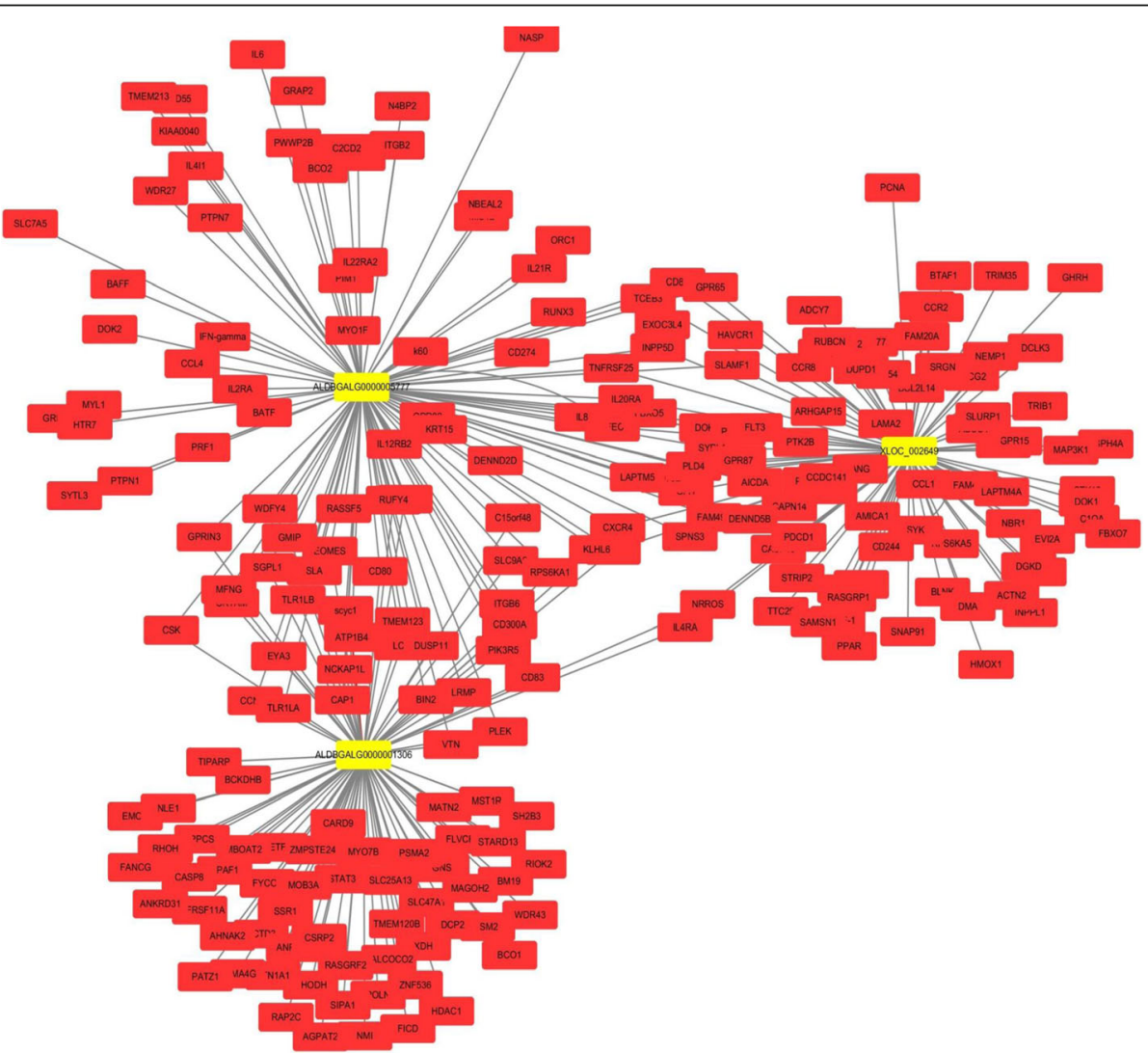

Fig. 7 Co-expression network of the representative IncRNAs and their partial target mRNAs. The relationships of mRNA-IncRNA were re-constructed based on the expression correlation coefficients (Pearson correlation $>0.95$ or $<-0.95$ ) using the cytoscape software (v3.6.0). Different colors were used to show different genes, with yellow for IncRNAs and red for mRNAs 


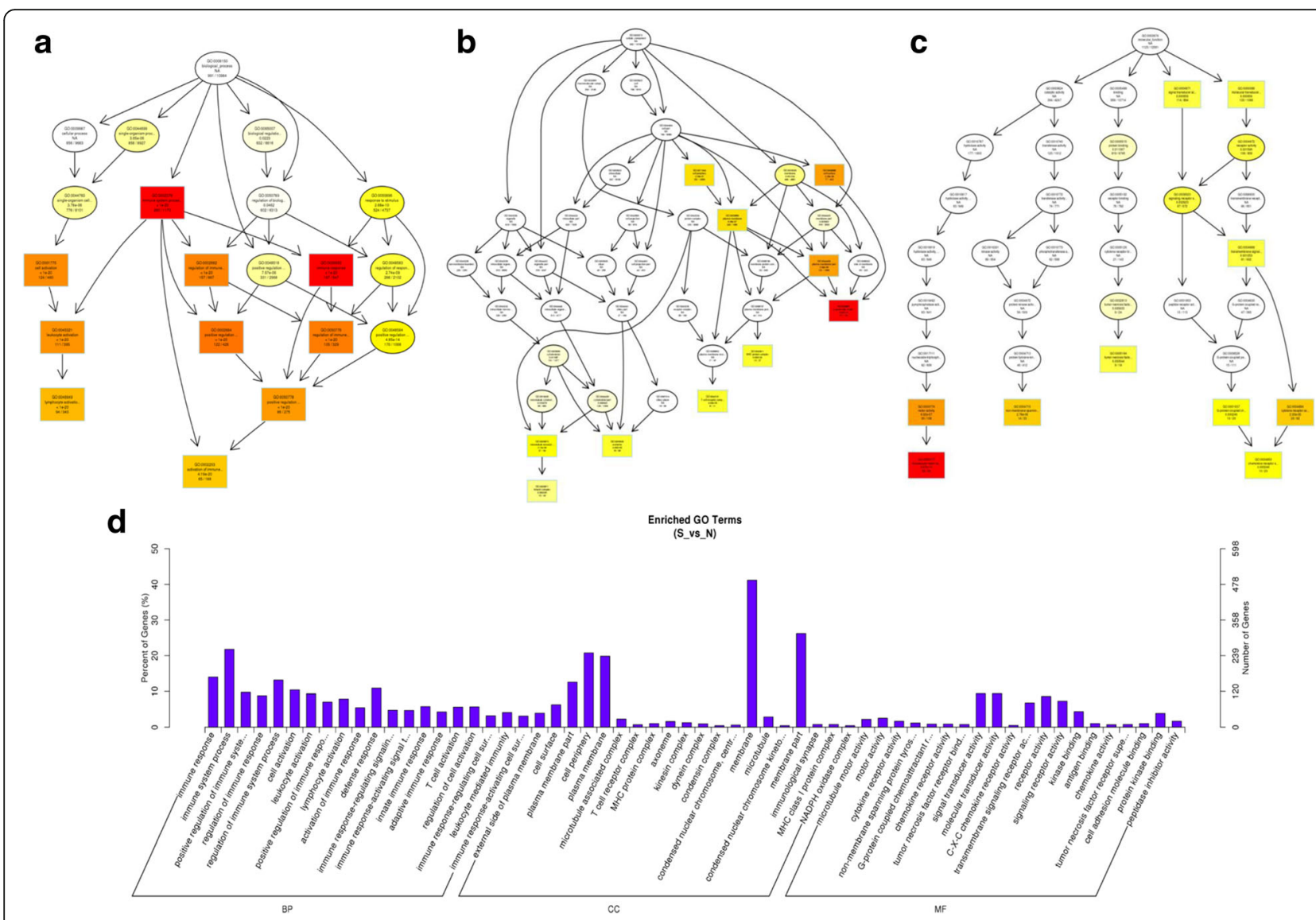

Fig. $8 \mathrm{GO}$ analysis of mRNAs in trachea tissue of chickens. The significant enriched biological process, cellular component and molecular function with changed mRNAs in trachea tissue of chicken were displayed. Directed Acyclic Graph (DAG) is the graphical display of GO enrichment results with candidate targeted genes (a-c). The number of genes in GO term were showed in histograph (d)

The pathways of cytokine-cytokine receptor interaction, CAMs and toll-like receptor signaling were significantly enriched by GO and KEGG enrichment analyses of these differentially expressed mRNAs, and previous studies also indicated that the infection of zoonotic C. parvum was closely associated with these pathways in the epithelial cells [59-61], indicating that these dysregulated genes may be involved in the interactions between the host and C. baileyi.

The potential functions of lncRNAs were commonly predicted by their target genes. In our study, the relationships between IncRNAs and their target genes colocated (within $100 \mathrm{~kb}$ ) and co-expressed (the Pearson's correlation coefficients $>0.95$ or $<-0.95$ ) were analyzed (Additional file 3, Fig. 7). Analyzing potential target genes of dysregulated lncRNAs revealed their important roles in regulation of the interaction between chickens and $C$. baileyi. For example, one lncRNA, ALDBGALG0000001306, was significantly upregulated between the infected and non-infected chickens, and its potential targets IL-6, IL-12 and IL-17 have been identified as important cytokines against Cryptosporidium infection [53, 62-64], suggesting that this lncRNA would be a regulator of immune response against $C$. baileyi infection in chickens.

Additionally, circRNAs, a newly discovered class of ncRNAs, may act as miRNA sponges to regulate the activity of target genes and participates in the regulation of gene transcription and protein production $[65,66]$ to affect prognosis of diseases, especially tumors [47, 49]. Therefore, circRNAs were tested in our study using RNA sequencing and only 104 circRNAs genes (65 were upregulated and 39 were downregulated) were demonstrated to be differentially expressed in response to the infection of $C$. baileyi. Compared with mRNAs and lncRNAs, the expression levels of circRNAs were generally lower (Fig. 6) and no significant GO terms were enriched in our study. The reasons for these differences may be due to the drawbacks of RNA sequencing protocol in our study or the expression of circRNA might be closely related to immune-specific organs and tissues (eg. brain, stem cells, testis and discs) predicted in previous studies [67-69]. The source genes of these circRNAs were mainly enriched in the metabolic pathway of amino 


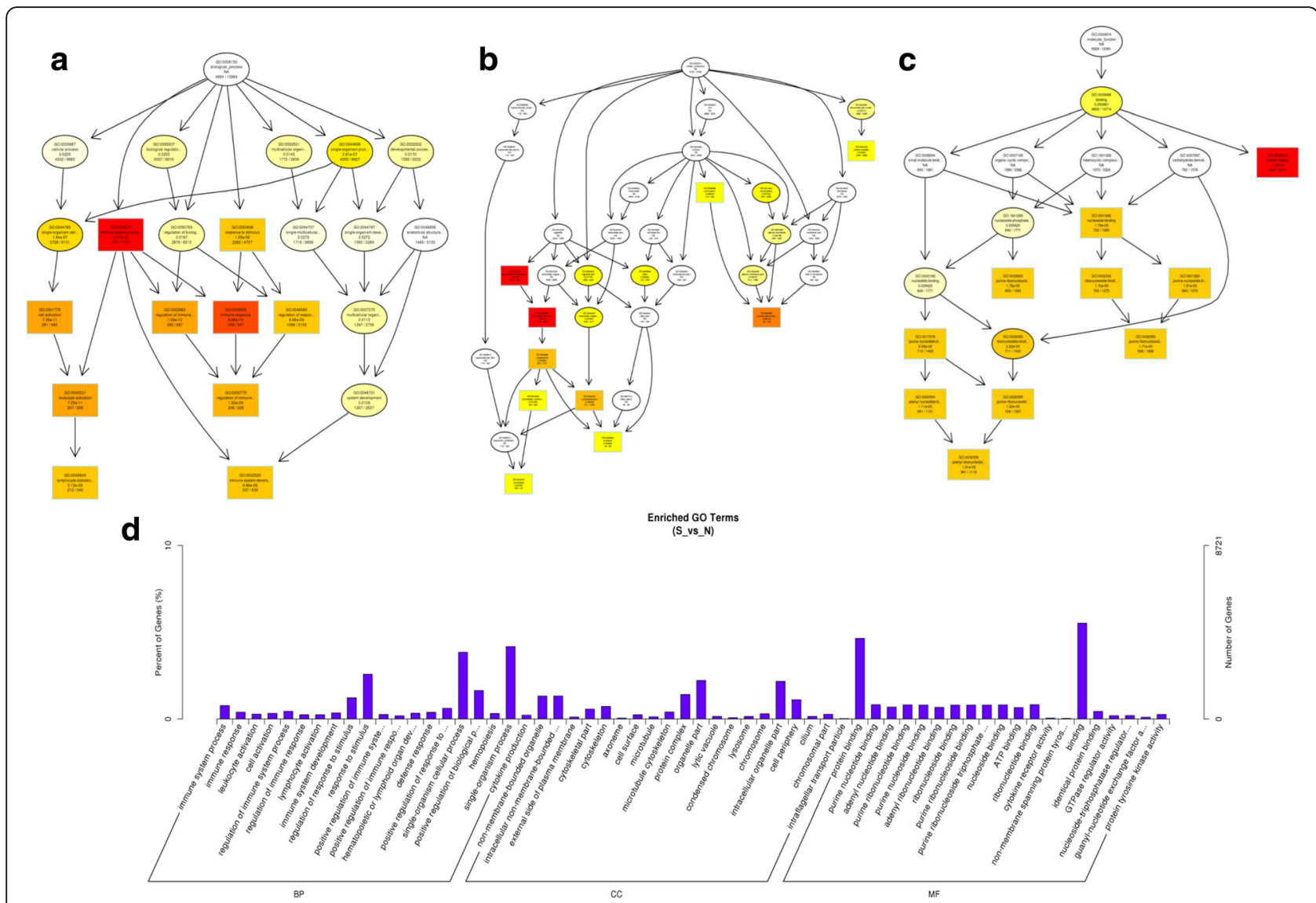

Fig. $9 \mathrm{GO}$ analysis of IncRNAs in trachea tissue of chickens. The significant enriched biological process, cellular component and molecular function with changed IncRNAs in trachea tissue of chicken were shown. Directed Acyclic Graph (DAG) is the graphical display of GO enrichment results with candidate targeted genes. The branch represents the relationship of inclusion, which defines the scope from increasingly small from top to bottom. The color depth represents the enrichment degree (a-c). The number of genes in $\mathrm{GO}$ term were showed in histograph (d)

sugar and nucleotide sugar metabolism, tight junction and glycerolipid metabolism. Some miRNAs (eg. let-7d, let-7f and let-7i) have been proven to regulate the expression of toll-like receptor four signal in respond to C. parvum infection $[25,26,70,71]$, and their interaction circRNAs (Additional file 4) were also found to be differentially expressed, suggesting that these circRNAs may implicate in the pathogenesis and progression of C. baileyi infection.

\section{Conclusions}

Differentially expressed mRNAs, IncRNAs and circRNAs were screened from chickens after C. baileyi infection. A total of 1317 mRNAs, 124 lncRNAs and 104 circRNAs were differentially expressed, and these RNAs could regulate expression of their related genes and play a key role in the pathogenesis of C. baileyi infection. The relevant signaling pathway of these predicted mRNAs and ncRNAs would be the focus of future study to fully reveal the pathogenesis of $C$. baileyi infection. The current work also provides new insight into the pathways and mechanisms that mediate the host immune response to C. baileyi (albeit in birds only).

\section{Methods}

\section{C. baileyi infection model}

A total of six newly hatched chickens were purchased from the Giant Long Company (Shaanxi, China), and reared in a pathogen-free laboratory and given constant light and adequate food and water during the entire experimental period. Chickens were divided into two groups randomly with three chickens per group and each chicken was kept in a separate cage. After normal feeding for 3 days, each chicken in the experimental group (S group) was orally infected with $1 \times 10^{6} \mathrm{C}$. baileyi oocysts, while the control group (N group) was given the same volume of PBS. Faecal samples were collected daily post infection (pi). The hemacytometer was used to record the numbers of oocyst per gram (OPG) of faeces. The successful infection was confirmed by the oocyst excretion (Additional file 7) and parasites in 


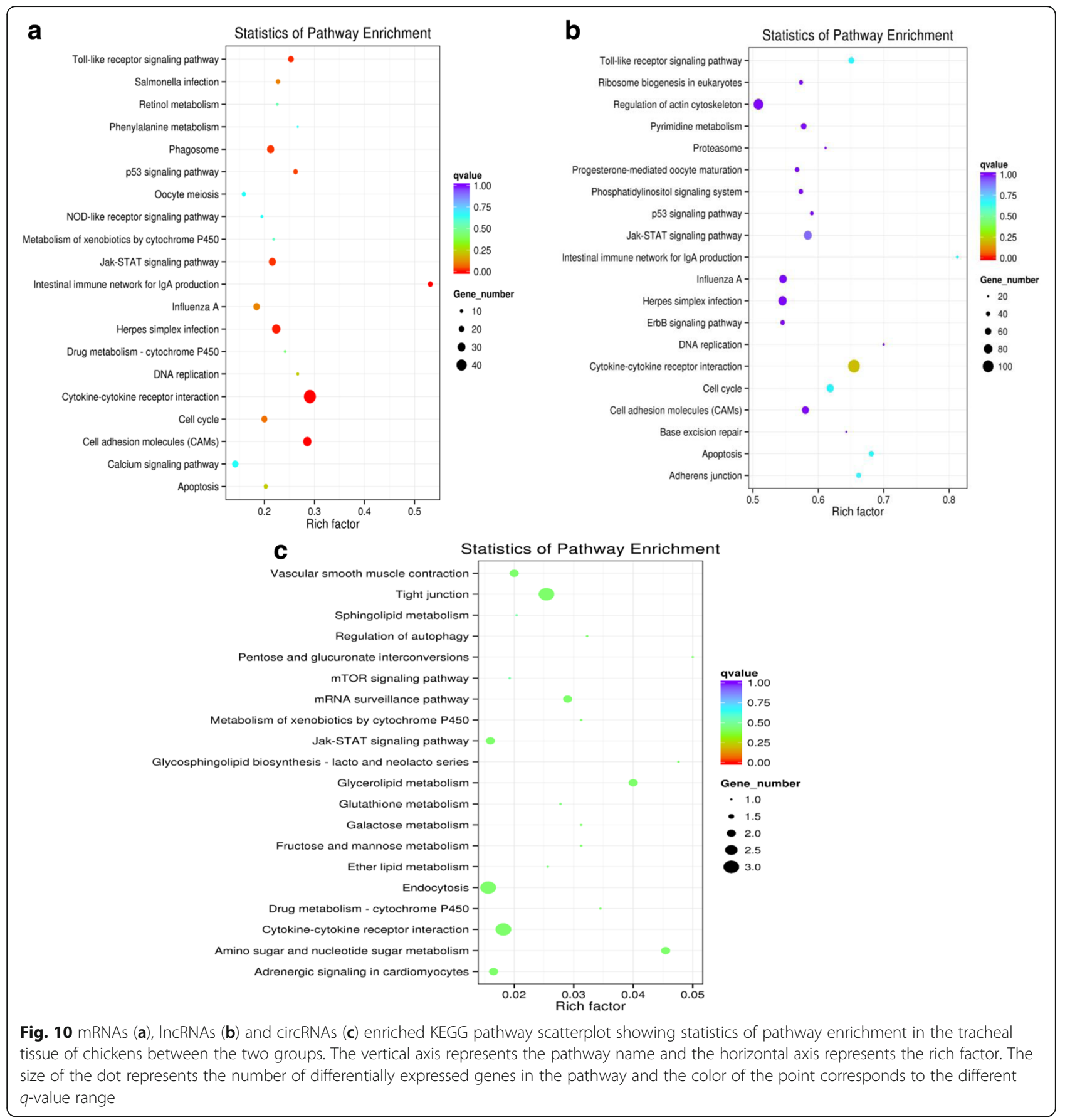

histological observations by haemotoxylin \& eosinstaining and by electron microscopy (Additional file 8).

\section{Sample collection and preparation}

Since $C$. baileyi mainly parasitizes in the respiratory tract, causing a series of respiratory diseases, at the first peak of oocyst excretion $(10 \mathrm{dpi})$, the tracheas of three chickens in both experimental and control groups were collected and sent to the company (Novogene, Beijing) for sequencing. Chickens were firstly kept into inverted beakers with infiltrated ether ball for several minutes. When they presented symptoms of slow breathing, absent corneal reflexes and skin sensation, these animals were then anesthetized. The trachea from each chicken was rapidly sampled and washed with nuclease-free PBS. All experiments were permitted by ethics committee of Northwest A\&F University and conducted in the fume hood. All the instruments and reagents were treated with DEPC in advance and without RNase. 
RNA isolation, library preparation and sequencing

Total RNA was extracted from each trachea sample using TRIzol reagent (Invitrogen, Carlsbad, CA, USA) according to the manufacturer's instructions. The concentration and purity of RNA were examined using the Qubit $^{\oplus}$ RNA Assay Kit in Qubit $^{\odot} 2.0$ Flurometer (Life Technologies, CA, USA) and the NanoPhotometer ${ }^{\bullet}$ spectrophotometer (IMPLEN, CA, USA), respectively. The RNA integrity was measured using the RNA Nano 6000 Assay Kit of the Bioanalyzer 2100 system (Agilent Technologies, CA, USA). A total amount of $3 \mu \mathrm{g}$ RNA per sample was then used for RNA sequencing. Firstly, the ribosomal RNA (rRNA) was removed by Epicentre Ribo-zero $^{\mathrm{Tm}}$ rRNA Removal Kit (Epicentre, USA). Subsequently, the RNAs were subjected to generate sequencing libraries by NEBNext $^{\ominus}$ Ultra $^{\text {тм }}$ Directional RNA Library Prep Kit (NEB, USA). After that, the first and second strand of the complementary DNA (cDNA) were synthesized and the AMPure XP system (Beckman Coulter, Beverly, USA) was used to purify the library fragments for firstly selecting cDNA fragments with a length of 150-200 bp. PCR was then performed and the Agilent Bioanalyzer 2100 system was used to evaluate the quality of the library. HiSeq PE Cluster Kit v4 cBot (Illumina) was used to perform the clustering of indexcoded samples according to the manufacturer's instructions. The libraries were sequenced on an Illumina Hiseq 4000 platform and $150 \mathrm{bp}$ paired-end reads were produced after cluster generation. For circRNA sequencing, the RNase R (Epicentre, USA) was additionally used to deal with the rRNA-depleted RNAs and remove the linear RNAs before sequencing library generation.

\section{Quality control}

The raw reads were processed firstly by the in-house perl scripts. To obtain clean reads, low quality reads, containing adapter and ploy- $\mathrm{N}$ reads were removed from raw data in this step. Meanwhile, the GC content, Q20 and Q30 of the clean data were also calculated. The clean data with high quality was used for further analysis.

\section{Mapping to the reference genome}

The reference genome (ftp://ftp.ensembl.org/pub/release-83/fasta/gallus_gallus/dna/) and gene model annotation files (ftp://ftp.ensembl.org/pub/release-83/gtf/ gallus_gallus/) were downloaded from website directly. Bowtie (v2.0.6) [72] was used to build index of the reference genome, and TopHat (v2.0.9) [73] was used to align paired-end clean reads to the reference genome. In addition, the software find_circ [74] was used to extend the anchor sequences and the back-spliced reads containing at least two supporting reads were considered to be circRNAs.

\section{Transcriptome assembly of IncRNAs}

The softwares Scripture beta2 [75] and Cufflinks (v2.1.1) $[76,77]$ were used to assemble mapped reads of each sample. These two methods determined exons connectivity by using spliced reads in different ways. With setting other parameters as default, Scripture and Cufflinks were run with 'min-frags-per-transfrag $=0$ ' and ‘-library-type', respectively.

\section{LncRNAs coding potential and conservation analysis}

Four analytic tools, namely coding-non-coding-index (CNCI) [78], coding potential calculator (CPC) [79], phylogenetic codon substitution frequency (PhyloCSF) [80] and Pfam-scan [81], were used to effectively distinguish protein-coding and non-coding sequences. Ultimately, the transcripts predicted with coding potential by any of the four tools were filtered out, and those without coding potential would to be our candidate set of lncRNAs.

The software phast (v1.3) [82] was usually used for phylogenetic analysis to evaluate the sequence conservation of transcripts. Then, we used the program phyloFit to calculate phylogenetic models of the conserved and non-conserved regions among species. PhastCons was used to compute the conservation scores of coding genes and lncRNAs.

\section{Target gene prediction and differential expression analysis}

Both cis and trans roles of target genes for lncRNA were predicted. Here, we searched coding genes within $100 \mathrm{~kb}$ upstream and downstream of each lncRNA and then analyzed their function. Fragments per kilobase for a million reads (FPKMs) of both lncRNAs and coding genes in each sample [77] were calculated by the Cuffdiff (v2.1.1). Unlike the former, psRobot [83] was used to predict the miRNA binding sites of circRNAs. Besides, the transcript per million (TPM) was used to normalize the expression level of circRNAs according to the criteria described by Zhou et al. [84]. Between two groups of chickens, transcripts or genes with $p$-value adjusted by the Benjamini \& Hochberg method ( $q$-value) $<0.05$ were considered significant.

\section{GO and KEGG enrichment analysis}

To explore the function of mRNAs, lncRNAs and circRNAs, the Gene Ontology (GO) and Kyoko Encyclopedia of Genes and Genomes (KEGG) enrichment analysis were conducted. The GOseq (v1.18.0) was used to perform the GO enrichment analysis of differentially expressed genes or target genes of lncRNAs or source genes of differentially expressed circRNAs, in which gene length bias was corrected. All three Go categories, namely cellular component, biological process, and molecular function, were included, and GO terms with the $q$-value $<0.05$ was 
considered significantly enriched. KOBAS [51] software was used to examine the statistical enrichment analysis of differential expression genes or lncRNA target genes or source genes of differentially expressed circRNAs in KEGG pathways. The gene sets were firstly mapped to database genes, and then compared with reference chicken genome (ftp://ftp.ensembl.org/pub/release-83/ fasta/gallus_gallus/dna/) to scan enriched pathways, diseases and functions. The enriched information was then evaluated by the statistical test and correction. The EASE score was calculated to test the relevance, and $p$-value $<0$. 05 was considered significantly enriched by differentially expressed genes.

\section{Validation by quantitative real-time polymerase chain reaction (qRT-PCR)}

Three trachea RNA samples from both infected and non-infected chickens were analyzed by qRT-PCR. Total cDNA of each sample was synthesized using two-step reverse transcriptase Kit (Vazyme Biotech Co., Ltd., Nanjing, China) according to the manufacturer's instructions. qRT-PCR were performed using LightCycler 480II Real-Time PCR System (Roche, Indianapolis, Indiana) and UltraSYBR Mixture (Qiagen, Shanghai, China). Each reaction (in $10 \mu \mathrm{L}$ ) contained $5 \mu \mathrm{L} 2 \times$ QuantiFast $^{\circ}$ SYBR $^{\circ}$ Green PCR Master Mix, $0.4 \mu \mathrm{L}$ primers $(5 \mu \mathrm{M})$ (Additional file 9), and $1 \mu \mathrm{L}$ cDNA. The protocol included an initial single cycle denaturing at $95{ }^{\circ} \mathrm{C}$ for $10 \mathrm{~min}$, followed by 40 cycles of denaturing at $95^{\circ} \mathrm{C}$ for $10 \mathrm{~s}$ and annealing at $60{ }^{\circ} \mathrm{C}$ or $55{ }^{\circ} \mathrm{C}$ (Additional file 9) for $30 \mathrm{~s}$. All amplifications were followed by dissociation curve analysis of the amplified products. Specific primers were designed using the Primer Premier (v5.0), and specificities were confirmed with BLAST. The gene expression levels were normalized with the reference gene $G A P D H$ by using $2^{-\triangle \triangle C t}$ value methods. The data were presented as the means \pm SEM.

\section{Statistical analysis}

The Pearson's correlation coefficients were used to calculate expression correlation between lncRNAs and mRNAs $(r>0.95$ or $r<-0.95)$. The statistical difference in gene expression of qRT-PCR results was analyzed by Student's t-test and false discovery rate (FDR) was also calculated to correct the $p$-value. It was considered to be statistically significant when $p$-value $<0.05$ or $q$-value $<0.05$.

\section{Additional files}

Additional file 1: The information of all novel IncRNAs identified in this study. (XLSX $106 \mathrm{~kb}$ )

Additional file 2: All the differentially expressed mRNAs, IncRNAs and circRNAs in this study. (XLSX $150 \mathrm{~kb}$ )
Additional file 3: LncRNA-protein coding gene pairs with co-location and co-expression relationships. (XLSX $13272 \mathrm{~kb})$

Additional file 4: The results of circRNA-binding miRNAs analysis. (XLSX $33940 \mathrm{~kb}$ )

Additional file 5: $\mathrm{GO}$ enrichment analysis of mRNAs and IncRNAs. (XLS $18518 \mathrm{~kb}$ )

Additional file 6: KEGG pathway analysis of mRNAs, IncRNAs and circRNAs. (XLS $255 \mathrm{~kb})$

Additional file 7: The oocyst excretion of chickens infected with $C$. baileyi. The horizontal axis represents the day post infection (dpi) with $C$. baileyi oocysts of chickens and the vertical axis shows the number of OPG. The oocysts were detected in faeces of infected chickens from 5 $\mathrm{dpi}$, and the peaks of oocyst excretion were observed at 10 and $16 \mathrm{dpi}$. (TIF $6095 \mathrm{~kb}$ )

Additional file 8: The parasites in histological observations by haemotoxylin \& eosin staining (A and B) and by electron microscopy ( $C$ and $D)$. No parasite was detected in the tracheas of the control chickens with haemotoxylin \& eosinstaining $(A, \times 400)$ and electron microscopy $(C, \times 2000)$, but there were a lot of parasites in the tracheas of the experimental chickens (B and D). (TIF $4763 \mathrm{~kb}$ )

Additional file 9: Primers used in qRT-PCR analysis. (XLSX $10 \mathrm{~kb}$ )

\section{Abbreviations}

BP: Biological process; C. baileyi: Cryptosporidium baileyi; C. parvum: Cryptosporidium parvum; CAMs: Cell adhesion molecules; CC: Cellular component; CDNA: Complementary DNA; circRNAs: Circular RNAs; CNCI: Coding-non-coding-index; CPC: Coding potential calculator; DAG: Directed acyclic graph; FDR: False discovery rate; FPKMs: Fragments per kilobase for a million reads; GO: Gene ontology; KEGG: Kyoto Encyclopedia of Genes and Genomes; lincRNAs: Long intergenic non-coding RNAs;

IncRNAs: Long non-coding RNAs; MF: Molecular function; miRNA: microRNA; ncRNA: Non-coding RNA; OPG: Oocyst per gram; ORF: Open reading frame; PhyloCSF: Phylogenetic codon substitution frequency; pi: Post infection; qRTPCR: Quantitative real-time polymerase chain reaction; RNA-seq: RNA sequencing; rRNA: Ribosomal RNA; SEM: Standard error of mean; TPM: Transcript per million

\section{Acknowledgements}

We thank Prof. Xian-Ming Chen from Creighton University and Prof. Xing-Quan Zhu from Lanzhou Veterinary Research Institute, Chinese Academy of Agricultural Sciences for their assistance in reviewing and copy-editing the language of this article.

\section{Funding}

This work was supported by National Key Research and Development Program of China (Grant number 2017YFD0501305), National Natural Science Foundation of China (Grant number 31572509), Shaanxi Science and Technology Project (Grant number 2016NY-113), China Postdoctoral Science Foundation funded project (Grant number 2016 M592848), and Shaanxi Postdoctoral Science Foundation.

\section{Availability of data and materials \\ We promise that all the data supporting the set of our conclusions are included in this article and its additional files. The complete RNA sequencing data generated and analyzed in this study have been submitted to the Genome Expression Omnibus (GEO) database, and the accession number is GSE108147 (https://www.ncbi.nlm.nih.gov/geo/query/ acc.cgi?acc $=$ GSE108147).}

\section{Authors' contributions}

The experiments were conceived and designed by GHZ. GJR and XCF established the C. baileyi infection model. The samples were collected and prepared by GJR, XCF, TLL and SSW. GJR conducted the data analysis and qRT-PCR validation, and wrote the manuscript under the help and guidance of GHZ. All authors have read and approved the final manuscript. 


\section{Ethics approval}

This study was carried out strictly in accordance with the recommendations in the Guide for the Care and Use of Laboratory Animals of the Ministry of Health, China. Our protocol with all animal experiments was approved by the Research Ethics Committee of Northwest A\&F University and they agreed to anesthetise animals with the ether that was used for rats in previous studies. All efforts were made to minimize the pain of animals used in this study.

\section{Competing interests}

The authors declare that they have no competing interests.

\section{Publisher's Note}

Springer Nature remains neutral with regard to jurisdictional claims in published maps and institutional affiliations.

\section{Received: 18 December 2017 Accepted: 2 May 2018 Published online: 10 May 2018}

\section{References}

1. Amer S, Wang $\mathrm{C}, \mathrm{He} \mathrm{H}$. First detection of Cryptosporidium baileyi in Ruddy Shelduck (Tadorna ferruginea) in China. J Vet Med Sci. 2010;72(7):935-8.

2. Qi M, Wang R, Ning C, Li X, Zhang L, Jian F, Sun Y, Xiao L. Cryptosporidium spp. in pet birds: genetic diversity and potential public health significance. Exp Parasitol. 2011:128(4):336-40.

3. Slavin D. Cryptosporidium meleagridis (sp. nov.). J Comp Pathol. 1955;65(3): 262-6.

4. Current WL, Upton SJ, Haynes TB. The life cycle of Cryptosporidium baileyi $\mathrm{n}$. Sp. (Apicomplexa, Cryptosporidiidae) infecting chickens. J Protozool. 1986;33(2):289-96.

5. Ryan UM, Xiao L, Read C, Sulaiman IM, Monis P, Lal AA, Fayer R, Pavlasek I. A redescription of Cryptosporidium galli Pavlásek, 1999 (Apicomplexa: Cryptosporidiidae) from birds. J Parasitol. 2003;89(4):809-13.

6. Holubová N, Sak B, Horčičková M, Hlásková L, Květoňová D, Menchaca S, McEvoy J, Kváč M. Cryptosporidium avium n. sp. (Apicomplexa: Cryptosporidiidae) in birds. Parasitol Res. 2016:115(6):2243-51.

7. Nakamura AA, Meireles MV. Cryptosporidium infections in birds - a review. Rev Bras Parasitol Vet. 2015;24(3):253-67.

8. Chelladurai JJ, Clark ME, Kváč M, Holubová N, Khan E, Stenger BL, Giddings CW, McEvoy J. Cryptosporidium galli and novel Cryptosporidium avian genotype $\mathrm{VI}$ in North American red-winged blackbirds (Agelaius phoeniceus). Parasitol Res. 2016;115(5):1901-6.

9. Huber F, da Silva S, Bomfim TC, Teixeira KR, Bello AR. Genotypic characterization and phylogenetic analysis of Cryptosporidium sp. from domestic animals in Brazil. Vet Parasitol. 2007;150(1-2):65-74.

10. Ryan U. Cryptosporidium in birds, fish and amphibians. Exp Parasitol. 2010;124(1):113-20.

11. Molina-Lopez RA, Ramis A, Martin-Vazquez S, Gomez-Couso H, Ares-Mazas E, Caccio SM, Leiva M, Darwich L. Cryptosporidium baileyi infection associated with an outbreak of ocular and respiratory disease in otus owls (Otus scops) in a rehabilitation Centre. Avian Pathol. 2010;39(3):171-6.

12. Abbassi H, Coudert F, Chérel Y, Dambrine G, Brugère-Picoux J, Naciri M. Renal cryptosporidiosis (Cryptosporidium baileyi) in specific-pathogen-free chickens experimentally coinfected with Marek's disease virus. Avian Dis. 1999;43(4):738-44.

13. Blagburn BL, Lindsay DS, Hoerr FJ, Davis JF, Giambrone JJ. Pathobiology of cryptosporidiosis (C. baileyi) in broiler chickens. J Protozool. 1991;38(6):25S-8S.

14. Goodwin MA, Brown J. Effect of Cryptosporidium baileyi on broilers infected at 26 days of age. Avian Dis. 1990;34(2):458-62.

15. Ditrich O, Palkovic L, Stĕrba J, Prokopic J, Loudová J, Giboda M. The first finding of Cryptosporidium baileyi in man. Parasitol Res. 1991;77(1):44-7.

16. Cabada MM, White AC Jr. Treatment of cryptosporidiosis: do we know what we think we know? Curr Opin Infect Dis. 2010;23(5):494-9.

17. Bonnin A, Lapillonne A, Petrella T, Lopez J, Chaponnier C, Gabbiani G, Robine S, Dubremetz JF. Immunodetection of the microvillous cytoskeleton molecules villin and ezrin in the parasitophorous vacuole wall of Cryptosporidium parvum (Protozoa: Apicomplexa). Eur J Cell Biol. 1999;78(11):794-801.

18. De Souza W. Microscopy and cytochemistry of the biogenesis of the parasitophorous vacuole. Histochem Cell Biol. 2005;123(1):1-18.
19. Huang BQ, Chen XM, LaRusso NF. Cryptosporidium parvum attachment to and internalization by human biliary epithelia in vitro: a morphologic study. J Parasitol. 2004;90(2):212-21.

20. Zhang H, Guo F, Zhu G. Cryptosporidium lactate dehydrogenase is associated with the Parasitophorous vacuole membrane and is a potentia target for developing therapeutics. PLoS Pathog. 2015;11(11):e1005250.

21. Sam-Yellowe TY. Rhoptry organelles of the apicomplexa: their role in host cell invasion and intracellular survival. Parasitol Today. 1996;12(8):308-16.

22. Valentini E, Cherchi S, Possenti A, Dubremetz JF, Pozio E, Spano F. Molecular characterisation of a Cryptosporidium parvum rhoptry protein candidate related to the rhoptry neck proteins TgRON1 of Toxoplasma gondii and PfASP of Plasmodium falciparum. Mol Biochem Parasitol. 2012;183(1):94-9.

23. Mauzy MJ, Enomoto S, Lancto CA, Abrahamsen MS, Rutherford MS. The Cryptosporidium parvum transcriptome during in vitro development. PLoS One. 2012;7(3):e31715.

24. Yang YL, Serrano MG, Sheoran AS, Manque PA, Buck GA, Widmer G. Overexpression and localization of a host protein on the membrane of Cryptosporidium parvum infected epithelial cells. Mol Biochem Parasitol. 2009:168(1):95-101.

25. Zhou R, Hu G, Liu J, Gong AY, Drescher KM, Chen XM. NF-kappaB p65dependent transactivation of miRNA genes following Cryptosporidium parvum infection stimulates epithelial cell immune responses. PLoS Pathog. 2009;5(12):e1000681.

26. Zhou R, Feng Y, Chen XM. Non-coding RNAs in epithelial immunity to Cryptosporidium infection. Parasitology. 2014;141(10):1233-43.

27. Bhan A, Mandal SS. Long noncoding RNAs: emerging stars in gene regulation, epigenetics and human disease. ChemMedChem. 2014:9(9):1932-56.

28. Qu S, Yang X, Li X, Wang J, Gao Y, Shang R, Sun W, Dou K, Li H. Circular RNA: a new star of noncoding RNAs. Cancer Lett. 2015;365(2):141-8.

29. Hirata H, Hinoda Y, Shahryari V, Deng G, Nakajima K, Tabatabai ZL, Ishii N, Dahiya R. Long noncoding RNA MALAT1 promotes aggressive renal cell carcinoma through Ezh2 and interacts with miR-205. Cancer Res. 2015;75(7): 1322-31.

30. Liu B, Sun L, Liu Q, Gong C, Yao Y, Lv X, Lin L, Yao H, Su F, Li D, Zeng M, Song E. A cytoplasmic NF-kB interacting long noncoding RNA blocks IKB phosphorylation and suppresses breast cancer metastasis. Cancer Cell. 2015; 27(3):370-81.

31. Yuan JH, Yang F, Wang F, Ma JZ, Guo YJ, Tao QF, Liu F, Pan W, Wang $\Pi$, Zhou CC, Wang SB, Wang YZ, Yang $Y$, Yang $N$, Zhou WP, Yang GS, Sun $\mathrm{SH}$. A long noncoding RNA activated by TGF- $\beta$ promotes the invasion-metastasis cascade in hepatocellular carcinoma. Cancer Cell. 2014;25(5):666-81.

32. Chen Z, Luo Y, Yang W, Ding L, Wang J, Tu J, Geng B, Cui Q, Yang J. Comparison analysis of dysregulated LncRNA profile in mouse plasma and liver after hepatic ischemia/reperfusion injury. PLoS One. 2015;10(7): e0133462.

33. Gupta RA, Shah N, Wang KC, Kim J, Horlings HM, Wong DJ, Tsai MC, Hung $T$, Argani $P$, Rinn JL, Wang $Y$, Brzoska P, Kong B, Li R, West RB, van de Vijver MJ, Sukumar S, Chang HY. Long non-coding RNA HOTAIR reprograms chromatin state to promote cancer metastasis. Nature. 2010;464(7291):1071-6.

34. Ma MZ, Chu BF, Zhang Y, Weng MZ, Qin YY, Gong W, Quan ZW. Long noncoding RNA CCAT1 promotes gallbladder cancer development via negative modulation of miRNA-218-5p. Cell Death Dis. 2015:6:e1583.

35. Shi Y, Liu Y, Wang J, Jie D, Yun T, Li W, Yan L, Wang K, Feng J. Downregulated long noncoding RNA BANCR promotes the proliferation of colorectal Cancer cells via downregulation of p21 expression. PLoS One. 2015;10(4):e0122679.

36. He Y, Meng XM, Huang C, Wu BM, Zhang L, Lv XW, Li J. Long noncoding RNAs: novel insights into hepatocellular carcinoma. Cancer Lett. 2014:344(1):20-7.

37. Lee S, Kopp F, Chang TC, Sataluri A, Chen B, Sivakumar S, Yu H, Xie Y, Mendell JT. Noncoding RNA NORAD regulates genomic stability by sequestering PUMILIO proteins. Cell. 2016;164(1-2):69-80.

38. Liu W, Ma C, Yang B, Yin C, Zhang B, Xiao Y. LncRNA Gm15290 sponges miR-27b to promote PPARY-induced fat deposition and contribute to body weight gain in mice. Biochem Biophys Res Commun. 2017:493(3):1168-75.

39. Yu SY, Dong B, Zhou SH, Tang L. LncRNA UCA1 modulates cardiomyocyte apoptosis by targeting miR-143 in myocardial ischemia-reperfusion injury. Int J Cardiol. 2017;247:31. 
40. Li Z, Rana TM. Decoding the noncoding: prospective of IncRNA-mediated innate immune regulation. RNA Biol. 2014;11(8):979-85.

41. Mirza AH, Kaur S, Brorsson CA, Pociot F. Effects of GWAS-associated genetic variants on IncRNAs within IBD and T1D candidate loci. PLoS One. 2014;9(8):e105723.

42. Yan B, Yao J, Liu JY, Li XM, Wang XQ, Li YJ, Tao ZF, Song YC, Chen Q, Jiang Q. IncRNA-MIAT regulates microvascular dysfunction by functioning as a competing endogenous RNA. Circ Res. 2015;116(7):1143-56.

43. Hansen TB, Jensen TI, Clausen BH, Bramsen JB, Finsen B, Damgaard CK, Kjems J. Natural RNA circles function as efficient microRNA sponges. Nature. 2013:495(7441):384-8.

44. Li H, Yang J, Wei X, Song C, Dong D, Huang Y, Lan X, Plath M, Lei C, Ma Y, Qi $X$, Bai $Y$, Chen $H$. CircFUT10 reduces proliferation and facilitates differentiation of myoblasts by sponging miR-133a. J Cell Physiol. 2018; 233(6):4643-51.

45. Wilusz JE, Sharp PA. Molecular biology. A circuitous route to noncoding RNA. Science. 2013;340(6131):440-1.

46. Deng T, Yang L, Zheng Z, Li Y, Ren W, Wu C, Guo L. Calcitonin gene-related peptide induces IL-6 expression in RAW264.7 macrophages mediated by mmu_circRNA_007893. Mol Med Rep. 2017;16(6):9367-74.

47. Kong Z, Wan X, Zhang Y, Zhang P, Zhang Y, Zhang X, Qi X, Wu H, Huang J, Li Y. Androgen-responsive circular RNA circSMARCA5 is up-regulated and promotes cell proliferation in prostate cancer. Biochem Biophys Res Commun. 2017:493(3):1217-23.

48. Lai Z, Yang Y, Yan Y, Li T, Li Y, Wang Z, Shen Z, Ye Y, Jiang K, Wang S. Analysis of co-expression networks for circular RNAs and mRNAs reveals that circular RNAs hsa_circ_0047905, hsa_circ_0138960 and hascircRNA7690-15 are candidate oncogenes in gastric cancer. Cell Cycle. 2017; 16(23):2301-11.

49. Cui X, Niu W, Kong L, He M, Jiang K, Chen S, Zhong A, Li W, Lu J, Zhang L. hsa_circRNA_103636: potential novel diagnostic and therapeutic biomarker in major depressive disorder. Biomark Med. 2016;10(9):943-52.

50. Zhou M, Zhao H, Wang Z, Cheng L, Yang L, Shi H, Yang H, Sun J. Identification and validation of potential prognostic IncRNA biomarkers for predicting survival in patients with multiple myeloma. J Exp Clin Cancer Res. 2015;34:102

51. Mao X, Cai T, Olyarchuk JG, Wei L. Automated genome annotation and pathway identification using the KEGG orthology (KO) as a controlled vocabulary. Bioinformatics. 2005;21(19):3787-93.

52. Kanehisa M, Araki M, Goto S, Hattori M, Hirakawa M, Itoh M, Katayama T, Kawashima S, Okuda S, Tokimatsu T, Yamanishi Y. KEGG for linking genomes to life and the environment. Nucleic Acids Res. 2008;36(Database issue):D480-4.

53. Zhao GH, Cheng WY, Wang W, Jia YQ, Fang YQ, Du SZ, Yu SK. The expression dynamics of $\mathrm{LL}-17$ and Th17 response relative cytokines in the trachea and spleen of chickens after infection with Cryptosporidium baileyi. Parasit Vectors. 2014;7:212.

54. Wang R, Jian F, Sun Y, Hu Q, Zhu J, Wang F, Ning C, Zhang L, Xiao L. Largescale survey of Cryptosporidium spp. in chickens and Pekin ducks (Anas platyrhynchos) in Henan, China: prevalence and molecular characterization. Avian Pathol. 2010;39(6):447-51.

55. Egyed Z, Sréter T, Széll Z, Beszteri B, Dobos-Kovács M, Márialigeti K, Cornelissen AW, Varga I. Polyphasic typing of Cryptosporidium baileyi: a suggested model for characterization of cryptosporidia. J Parasitol. 2002;88(2):237-43.

56. Huang $L$, Zhu H, Zhang $S$, Wang R, Liu L, Jian F, Ning C, Zhang L. An in vitro model of infection of chicken embryos by Cryptosporidium baileyi. Exp Parasitol. 2014;147:41-7.

57. International Chicken Genome Sequencing Consortium. Sequence and comparative analysis of the chicken genome provide unique perspectives on vertebrate evolution. Nature. 2004;432(7018):695-716.

58. Wong GK, Liu B, Wang J, Zhang Y, Yang X, Zhang Z, Meng Q, Zhou J, Li D, Zhang J, Ni P, Li S, Ran L, Li H, Zhang J, Li R, Li S, Zheng H, Lin W, Li G, Wang X, Zhao W, Li J, Ye C, Dai M, Ruan J, Zhou Y, Li Y, He X, Zhang Y, Wang J, Huang $X$, Tong W, Chen J, Ye J, Chen C, Wei N, Li G, Dong L, Lan F, Sun Y, Zhang Z, Yang Z, Yu Y, Huang Y, He D, Xi Y, Wei D, Qi Q, Li W, Shi J, Wang $M$, Xie F, Wang J, Zhang X, Wang P, Zhao Y, Li N, Yang N, Dong W, Hu S, Zeng C, Zheng W, Hao B, Hillier LW, Yang SP, Warren WC, Wilson RK, Brandström M, Ellegren $H_{1}$ Crooijmans RP, van der Poel JJ, Bovenhuis $H_{\text {, }}$ Groenen MA, Ovcharenko I, Gordon L, Stubbs L, Lucas S, Glavina T, Aerts A, Kaiser $P$, Rothwell L, Young JR, Rogers S, Walker BA, van Hateren A, Kaufman J, Bumstead N, Lamont SJ, Zhou H, Hocking PM, Morrice D, de Koning DJ,
Law A, Bartley N, Burt DW, Hunt H, Cheng HH, Gunnarsson U, Wahlberg P, Andersson L, Kindlund E, Tammi MT, Andersson B, Webber C, Ponting CP, Overton IM, Boardman PE, Tang H, Hubbard SJ, Wilson SA, Yu J, Wang J, Yang $\mathrm{H}$, International Chicken Polymorphism Map Consortium. A genetic variation map for chicken with 2.8 million single-nucleotide polymorphisms. Nature. 2004;432(7018):717-22.

59. Lean IS, McDonald V, Pollok RC. The role of cytokines in the pathogenesis of Cryptosporidium infection. Curr Opin Infect Dis. 2002;15(3):229-34.

60. McDonald V, Korbel DS, Barakat FM, Choudhry N, Petry F. Innate immune responses against Cryptosporidium parvum infection. Parasite Immunol. 2013;35(2):55-64.

61. Yang Z, Fu Y, Gong P, Zheng J, Liu L, Yu Y, Li J, Li H, Yang J, Zhang X. Bovine TLR2 and TLR4 mediate Cryptosporidium parvum recognition in bovine intestinal epithelial cells. Microb Pathog. 2015;85:29-34.

62. Ehigiator HN, Romagnoli P, Borgelt K, Fernandez M, McNair N, Secor WE, Mead JR. Mucosal cytokine and antigen-specific responses to Cryptosporidium parvum in IL-12p40 KO mice. Parasite Immunol. 2005;27(1-2):17-28.

63. Pagani M, Rossetti G, Panzeri I, de Candia P, Bonnal RJ, Rossi RL, Geginat J, Abrignani S. Role of microRNAs and long-non-coding RNAs in CD4(+) T-cell differentiation. Immunol Rev. 2013;253(1):82-96.

64. Petry F, Jakobi V, Tessema TS. Host immune response to Cryptosporidium parvum infection. Exp Parasitol. 2010;126(3):304-9.

65. Chen LL, Yang L. Regulation of circRNA biogenesis. RNA Biol. 2015;12(4):381-8.

66. Li J, Yang J, Zhou P, Le Y, Zhou C, Wang S, Xu D, Lin HK, Gong Z. Circular RNAs in cancer: novel insights into origins, properties, functions and implications. Am J Cancer Res. 2015;5(2):472-80.

67. Ma CJ, Liu X, Che L, Liu ZH, Samartzis D, Wang HQ. Stem cell therapies for intervertebral disc degeneration: immune privilege reinforcement by Fas/ FasL regulating machinery. Curr Stem Cell Res Ther. 2015;10(4):285-95.

68. Liu ZH, Sun Z, Wang HQ, Ge J, Jiang TS, Chen YF, Ma Y, Wang C, Hu S, Samartzis D, Luo ZJ. FasL expression on human nucleus pulposus cells contributes to the immune privilege of intervertebral disc by interacting with immunocytes. Int J Med Sci. 2013;10(8):1053-60.

69. Lan PH, Liu ZH, Pei YJ, Wu ZG, Yu Y, Yang YF, Liu X, Che L, Ma CJ, Xie YK, Hu QJ, Wan ZY, Wang HQ. Landscape of RNAs in human lumbar disc degeneration. Oncotarget. 2016;7(39):63166-76.

70. Chen XM, Splinter PL, O'Hara SP, LaRusso NF. A cellular micro-RNA, let-7i, regulates toll-like receptor 4 expression and contributes to cholangiocyte immune responses against Cryptosporidium parvum infection. J Biol Chem. 2007;282(39):28929-38.

71. Hu G, Gong AY, Roth AL, Huang BQ, Ward HD, Zhu G, Larusso NF, Hanson ND, Chen XM. Release of luminal exosomes contributes to TLR4-mediated epithelial antimicrobial defense. PLoS Pathog. 2013;9(4):e1003261.

72. Langmead B, Salzberg SL. Fast gapped-read alignment with bowtie 2. Nat Methods. 2012:9(4):357-9.

73. Kim D, Pertea G, Trapnell C, Pimentel H, Kelley R, Salzberg SL. TopHat2: accurate alignment of transcriptomes in the presence of insertions, deletions and gene fusions. Genome Biol. 2013;14(4):R36.

74. Memczak S, Jens M, Elefsinioti A, Torti F, Krueger J, Rybak A, Maier L, Mackowiak SD, Gregersen LH, Munschauer M, Loewer A, Ziebold U, Landthaler M, Kocks C, le Noble F, Rajewsky N. Circular RNAs are a large class of animal RNAs with regulatory potency. Nature. 2013:495(7441):333-8.

75. Guttman M, Garber M, Levin JZ, Donaghey J, Robinson J, Adiconis X, Fan L, Koziol MJ, Gnirke A, Nusbaum C, Rinn JL, Lander ES, Regev A. Ab initio reconstruction of cell type-specific transcriptomes in mouse reveals the conserved multi-exonic structure of lincRNAs. Nat Biotechnol. 2010:28(5):503-10.

76. Cabili MN, Trapnell C, Goff L, Koziol M, Tazon-Vega B, Regev A, Rinn JL. Integrative annotation of human large intergenic noncoding RNAs reveals global properties and specific subclasses. Genes Dev. 2011;25(18):1915-27.

77. Trapnell C, Williams BA, Pertea G, Mortazavi A, Kwan G, van Baren MJ, Salzberg SL, Wold BJ, Pachter L. Transcript assembly and quantification by RNA-seq reveals unannotated transcripts and isoform switching during cell differentiation. Nat Biotechnol. 2010;28(5):511-5.

78. Sun L, Luo H, Bu D, Zhao G, Yu K, Zhang C, Liu Y, Chen R, Zhao Y. Utilizing sequence intrinsic composition to classify protein-coding and long noncoding transcripts. Nucleic Acids Res. 2013;41(17):e166. 
79. Kong L, Zhang Y, Ye ZQ, Liu XQ, Zhao SQ, Wei L, Gao G. CPC: assess the protein-coding potential of transcripts using sequence features and support vector machine. Nucleic Acids Res. 2007;35(Web Server issue):W345-9.

80. Lin MF, Jungreis I, Kellis M. PhyloCSF: a comparative genomics method to distinguish protein coding and non-coding regions. Bioinformatics. 2011;27(13):i275-82.

81. Punta M, Coggill PC, Eberhardt RY, Mistry J, Tate J, Boursnell C, Pang N, Forslund K, Ceric G, Clements J, Heger A, Holm L, Sonnhammer EL, Eddy SR, Bateman A, Finn RD. The Pfam protein families database. Nucleic Acids Res. 2012;40(Database issue):D290-301.

82. Siepel A, Bejerano G, Pedersen JS, Hinrichs AS, Hou M, Rosenbloom K, Clawson H, Spieth J, Hillier LW, Richards S, Weinstock GM, Wilson RK, Gibbs RA, Kent WJ, Miller W, Haussler D. Evolutionarily conserved elements in vertebrate, insect, worm, and yeast genomes. Genome Res. 2005;15(8):1034-50.

83. Wu HJ, Ma YK, Chen T, Wang M, Wang XJ. PsRobot: a web-based plant small RNA meta-analysis toolbox. Nucleic Acids Res. 2012:40(Web Server issue):W22-8.

84. Zhou L, Chen J, Li Z, Li X, Hu X, Huang Y, Zhao X, Liang C, Wang Y, Sun L, Shi M, Xu X, Shen F, Chen M, Han Z, Peng Z, Zhai Q, Chen J, Zhang Z, Yang R, Ye J, Guan Z, Yang H, Gui Y, Wang J, Cai Z, Zhang X. Integrated profiling of microRNAs and mRNAs: microRNAs located on Xq27.3 associate with clear cell renal cell carcinoma. PLoS One. 2010:5(12):e15224.

Ready to submit your research? Choose BMC and benefit from:

- fast, convenient online submission

- thorough peer review by experienced researchers in your field

- rapid publication on acceptance

- support for research data, including large and complex data types

- gold Open Access which fosters wider collaboration and increased citations

- maximum visibility for your research: over $100 \mathrm{M}$ website views per year

At BMC, research is always in progress.

Learn more biomedcentral.com/submissions 